\title{
A New Method of Construction of Robust Second Order Slope Rotatable Designs Using Pairwise Balanced Designs
}

\author{
Bejjam Re. Victorbabu, Kottapalli Rajyalakshmi \\ Department of Statistics, Acharya Nagarjuna University, Guntur, India \\ Email: victorsugnanam@yahoo.co.in, Rajyalakshmi_kottapalli@yahoo.com
}

Received May 10, 2012; revised June 12, 2012; accepted June 28, 2012

\begin{abstract}
In this paper, a new method of construction of robust second order slope rotatable designs (RSOSRD) using pairwise balanced designs (PBD) is suggested and also obtained the variance of the estimated derivatives for the factors $6 \leq v \leq$ 15. It is shown that the new method sometimes leads to designs with less number of design points compared to designs constructed with the help of balanced incomplete block designs (BIBD).
\end{abstract}

Keywords: Response Surface Designs; Slope Rotatability; Correlated Errors; Robustness; Second Order Slope Rotatable Designs (SOSRD)

\section{Introduction}

In response surface methodology, rotatability is a natural and highly desirable property. This was introduced and developed by Box and Hunter [1] assuming the errors to be uncorrelated and homoscedastic. Das and Narasimham [2] constructed second order rotatable designs (SORD) through balanced incomplete block designs (BIBD). Tyagi [3] constructed SORD using pairwise balanced designs (PBD). Panda and Das [4] studied first order rotatable designs with correlated errors. In order to study the nature of robust rotatable designs, rotatability conditions for second order regression designs have been derived, assuming the errors to be correlated. These conditions have been further studied under different variance covariance structures of errors. Das [5,6] introduced robust second order rotatable designs (RSORD). Rajyalakshmi and Victorbabu [7] constructed robust rotatable central composite designs (RRCCD) for factors $2 \leq v \leq 17$. Victorbabu and Rajyalakshmi [8] constructed a new method of construction of robust second order rotatable designs using BIBD. Victorbabu and Rajyalakshmi [9] studied a new method of construction of robust second order rotatable designs using PBD.

In response surface methodology, good estimators of the derivatives of the response function may be as important or perhaps more important than estimation of mean response.

Estimation of differences in responses at two different points in the factor space will often be of great importance. If a difference in responses at two points close together is of interest then estimation of local slope (rate of change) of the response is required. Estimation of slopes occurs frequently in practical situations. For instance, there are cases in which we want to estimate rate of reaction in chemical experiment, rate of change in the yield of a crop to various fertilizer doses, rate of disintegration of radioactive material in animal etc. [9].

Murty and Studden [10] suggested optimal designs for estimating the slope of a polynomial regression. Hader and Park [11] introduced slope rotatable central composite designs assuming errors are uncorrelated and homoscedastic. Park [9] studied a class of multifactor designs for estimating the slope of response surfaces. Victorbabu and Narasimham [12] constructed second order slope rotatable designs (SOSRD) using BIBD assuming errors are uncorrelated and homoscedastic. Victorbabu and Narasimham [13] constructed SOSRD using PBD. Several authors have studied slope rotatable designs assuming errors to be uncorrelated and homoscedastic. However it is not uncommon to come across some practical situations when the errors are correlated, violating the usual assumptions. Specifically Das [14] introduced the concept of slope rotatability with correlated errors, which requires that the variance of the estimated derivative to be constant, independent of correlation parameter involved in the variance-covariance structure of errors. They have studied slope rotatability conditions for a second order design with correlated errors. Victorbabu and Rajyalakshmi [15] studied robust slope rotatable central composite designs (RSRCCD) for the factors $2 \leq$ $v \leq 8$. Further, Victorbabu and Rajyalakshmi [16] studied robust slope rotatable designs (RSOSRD) using BIBD for the factors $3 \leq v \leq 8$.

In this paper, an attempt is made to construct RSO- 
SRD using PBD and also obtained the variance of the estimated derivatives for factors $6 \leq v \leq 15$.

\section{Second Order Response Surface Designs with Correlated Errors}

Assuming that the response surface is of second order, we adopt the model:

$$
Y_{u}=\beta_{0}+\sum_{i=1}^{v} \beta_{i} x_{i u}+\sum_{i=1}^{v} \beta_{i i} x_{i u}^{2}+\sum \sum_{i \leq j=1}^{v} \beta_{i j} x_{i u} x_{j u}+e_{u}
$$

where $x_{\mathrm{iu}}$ denotes the level of the $i^{\text {th }}$ factor $(i=1,2, \cdots, v)$ in the $u^{\text {th }}$ run $(u=1,2, \cdots, N)$ of the experiment, $\mathrm{e}_{u}$ 's are correlated errors. Here $\beta_{0}, \beta_{i}, \beta_{i i}, \beta_{i j}$ are the parameters of the model and $Y_{u}$ is the observed response at the $u^{\text {th }}$ design point.

\section{Second Order Slope Rotatable Designs with Correlated Errors}

Following Hader and Park [11], Victorbabu and Narasimham [12], Das [14], the necessary and sufficient conditions for slope rotatability in second order model with correlated errors are as follows:

Conditions for Second Order Slope Rotatable Designs with Correlated Errors.

The estimated response at $x$ is given by

$$
\hat{y}_{x}=\hat{\beta}_{0}+\sum_{i=1}^{v} \hat{\beta}_{i} x_{i}+\sum_{i=1}^{v} \hat{\beta}_{i} x_{i}^{2}+\sum \sum_{i \leq j=1}^{v} \hat{\beta}_{i j} x_{i} x_{j}
$$

For the second order model as in (2.2), we have

$$
\frac{\partial \hat{y}_{x}}{\partial x_{i}}=\hat{\beta}_{i}+2 \hat{\beta}_{i i} x_{i} x_{j}+\sum_{j=1 ; j \neq 1}^{v} \hat{\beta}_{i j} x_{j}
$$

The variance of $\frac{\partial \hat{y}_{x}}{\partial x_{i}}$ is given by,

$$
\begin{aligned}
V\left(\frac{\partial \hat{y}_{x}}{\partial x_{i}}\right)= & V\left(\hat{\beta}_{i}\right)+4 x_{i}^{2} V\left(\hat{\beta}_{i i}\right)+4 x_{i} \operatorname{Cov}\left(\hat{\beta}_{i}, \hat{\beta}_{i i}\right) \\
& +\sum_{j=1 ; j \neq i}^{v} x_{j}^{2} V\left(\hat{\beta}_{i j}\right)+\sum_{j=1 s=1 ;} \sum_{j \neq s \neq i}^{v} x_{j} x_{s} \operatorname{Cov}\left(\hat{\beta}_{i j}, \hat{\beta}_{i s}\right) \\
& +2 \sum_{j=1 ; j \neq i}^{v} x_{j} \operatorname{Cov}\left(\hat{\beta}_{i}, \hat{\beta}_{i j}\right) \\
& +4 \sum_{j=1 ; j \neq i}^{v} x_{i} x_{j} \operatorname{Cov}\left(\hat{\beta}_{i i}, \hat{\beta}_{i j}\right) \\
V\left(\frac{\partial \hat{y}_{x}}{\partial x_{i}}\right)= & v^{i . i}+4 x_{i}^{2} v^{i i . i i}+4 x_{i} v^{i . i i} \\
& +\sum_{j=1 ; j \neq i}^{v} x_{j}^{2} j^{i j . i j}+\sum_{j=1 s=1} \sum_{j \neq s \neq i}^{v} x_{j} x_{s} v^{i j . j i s} \\
& +2 \sum_{j=1 ; j \neq i}^{v} x_{j} v^{i . i . j}+4 \sum_{j=1 ; j \neq i}^{v} x_{i} x_{j} v^{i i . i j}
\end{aligned}
$$

The variance of estimated first order derivative with respect to each independent variable $x_{\mathrm{i}}$ as in (2.4) to be a function of $d^{2}=\sum_{i=1}^{v} x_{i u}^{2}$ if and only if,

$$
\begin{aligned}
& \text { 1) } v^{i . i i}=0 ; 1 \leq i \leq v, v^{i . i j}=0 \text {; } \\
& 1 \leq i, j \leq v, i \neq j \\
& \text { 2) } v^{i j . i j^{\prime}}=0 ; 1 \leq i \neq j \neq j^{\prime} \leq v \text {, } \\
& v^{i i . i j}=0 ; 1 \leq i, j \leq v, i \neq j \\
& \text { 3) } v^{i . i}=\text { constant } 1 \leq i \leq v \text {, } \\
& \text { 4) } v^{i i . i i}=\text { constant } 1 \leq i \leq v \text {, } \\
& \text { 5) } v^{i j . i j}=\text { constant } 1 \leq i<j \leq v \text {, and } \\
& \text { 6) } v^{i i . i i}=\frac{1}{4} v^{i j . i j} ; 1 \leq i<j \leq v \text {. }
\end{aligned}
$$

The following are the equivalent conditions of (1) through (5) in (2.5) for slope rotatability in secondorrelated errors model (2.1).

$$
\begin{aligned}
& \text { 1)*: a) } v_{0 . j}=v_{0 . j l}=0 ; 1 \leq j \leq v ; \\
& \text { b) } v_{i . j}=0 ; 1 \leq i, j \leq v, i \neq j ; \\
& \text { c): i) } v_{i i . j}=0 ; 1 \leq i, j \leq v ; \\
& \text { ii) } v_{i . j l}=0 ; 1 \leq i, j<l \leq v ; \\
& \text { iii) } v_{i i . . j l}=0 ; 1 \leq i, j<l \leq v, \quad(j, l) \neq(i, i) ; \\
& \text { iv) } v_{i i . . j l}=0 ; 1 \leq i, l<j, t \leq v,(i, j) \neq(l, t) ;
\end{aligned}
$$

2)*: a) $v_{0 . j j}=$ constant $=a_{0}$, say; $1 \leq j \leq v$;

b) $v_{i . i}=$ constant $=\frac{1}{e}$, say; $1 \leq i \leq v$;

c) $v_{i i . i i}=$ constant $=\eta\left(\frac{2}{g}+f\right)$, say; $1 \leq i \leq v$;

3)*: a) $v_{i i . j j}=$ constant $=f ; 1 \leq i \neq j \leq v$;

b) $v_{i j . i j}=$ constant $=\frac{1}{g} ; 1 \leq i<j \leq v$.

Following (2.6), the necessary and sufficient conditions for second order slope rotatability under the intraclass structure after some simplifications turn out to be

4.

1)*: $\sum_{u=1}^{N} x_{i_{1} u}^{\alpha_{1}} x_{i_{2} u}^{\alpha_{2}} x_{i_{3} u}^{\alpha_{3}} x_{i_{4} u}^{\alpha_{4}}=0$; for any $\alpha_{i}$ odd and $\sum_{i=1}^{4} \alpha_{i} \leq$

$$
\begin{aligned}
& \text { 2)*: a) } \sum_{u=1}^{N} x_{i u}^{2}=\text { constant }, 1 \leq i \leq v ; \\
& \text { b) } \sum_{u=1}^{N} x_{i u}^{4}=\text { constant } 1 \leq i \leq v ; \\
& \text { 3)* } \sum_{u=1}^{N} x_{i u}^{2} x_{j u}^{2}=\text { constant }, 1 \leq i, j \leq v, i \neq j ;
\end{aligned}
$$

Using $\sum_{u=1}^{N} x_{i u}^{2}=N \lambda_{2}, 1 \leq i \leq v$ and $\sum_{u=1}^{N} x_{i u}^{2} x_{j u}^{2}=N \lambda_{4}$, 
$1 \leq i, j \leq v, i \neq j$, the second order slope rotatable design parameters under the intra-class structure are as follows:

1) $a_{0}=\frac{N \lambda_{2}}{\sigma^{2}\{1+(N-1) \rho\}}$;

2) $f=\frac{\{1+(N-1) \rho\} N \lambda_{4}-\rho N^{2} \lambda_{2}^{2}}{\sigma^{2}(1-\rho)\{1+(N-1) \rho\}}$;

3) $\frac{1}{e}=\frac{N \lambda_{2}}{\sigma^{2}(1-\rho)}$;

4) $\frac{1}{g}=\frac{N \lambda_{4}}{\sigma^{2}(1-\rho)}$;

5) $v_{00}=\frac{N}{\sigma^{2}\{1+(N-1) \rho\}}$;

6) $\eta\left(\frac{2}{g}+f\right)=\frac{\eta\{1+(N-1) \rho\} 3 N \lambda_{4}-\rho N^{2} \lambda_{2}^{2}}{\sigma^{2}(1-\rho)\{1+(N-1) \rho\}}$.

Note that if $\rho=0$, the conditions (2.7) and (2.8) reduces to

1): $\sum_{u=1}^{N} x_{i_{1} u}^{\alpha_{1}} x_{i_{2} u}^{\alpha_{2}} x_{i_{3} u}^{\alpha_{3}} x_{i_{4} u}^{\alpha_{4}}=0 ;$ for any $\alpha_{i}$ odd and $\sum_{i=1}^{4} \alpha_{i} \leq$ 4.

2): a) $\sum_{u=1}^{N} x_{i u}^{2}=$ constant $=N \lambda_{2}, 1 \leq i \leq v$;

b) $\sum_{u=1}^{N} x_{i u}^{4}=$ constant $=c N \lambda_{4}, 1 \leq i \leq v$;

3) $\sum_{u=1}^{N} x_{i u}^{2} x_{j u}^{2}=$ constant $=N \lambda_{4}, 1 \leq i, j \leq v, i \neq j$

where $c=3 \eta$, and $\lambda_{4}, \lambda_{2}, \eta$ are constants.

The variances and covariances of the estimated parameters of the model (2.1) under the slope rotatability are as follows:

1) $V\left(\hat{\beta}_{0}\right)=\frac{\eta\left(\frac{2}{g}+f\right)+(v-1) f}{T} ; 1 \leq i \leq v$;

2) $V\left(\hat{\beta}_{i}\right)=e ; 1 \leq i \leq v$;

3) $V\left(\hat{\beta}_{i j}\right)=g ; 1 \leq i<j \leq v$;

$\begin{aligned}5)^{*}: & \eta\left(\frac{2}{g}+f\right)\left[4 v_{00}-e v_{00} \eta\left(\frac{2}{g}+f\right)-g v_{00} f(v-1)+g v a_{0}^{2}+v_{00} f g\right]+v_{00} f\{4(v-2)+(v-1) f g\} \\ & -a_{0}^{2}\{4(v-1)+v f g\}=0 .\end{aligned}$

Using (2.8), the condition (2.14) simplifies to

4) $V\left(\hat{\beta}_{i i}\right)=\frac{v_{00}\left\{\eta\left(\frac{2}{g}+f\right)+(v-2) f\right\}-(v-1) a_{0}^{2}}{T\left\{\eta\left(\frac{2}{g}+f\right)-f\right\}}$,

$1 \leq i \leq v$

5) $\operatorname{Cov}\left(\hat{\beta}_{0}, \hat{\beta}_{i i}\right)=\frac{-a_{0}}{T} ; 1 \leq i \leq v$;

6) $\operatorname{Cov}\left(\hat{\beta}_{i i}, \hat{\beta}_{j j}\right)=\frac{\left(a_{0}^{2}-f v_{00}\right)}{T\left\{\eta\left(\frac{2}{g}+f\right)-f\right\}} ; 1 \leq i \neq j \leq v$

where

$$
T=\left[v_{00}\left\{\eta\left(\frac{2}{g}+f\right)+(v-1) f\right\}-v a_{0}^{2}\right]
$$

and other covariance's are zero.

An inspection of the variance of $\hat{\beta}_{0}$ shows that a necessary condition for the existence of a non-singular second order design is $T>0$ i.e.,

$$
4)^{*}:\left[v_{00}\left\{\eta\left(\frac{2}{g}+f\right)+(v-1) f\right\}-v a_{0}^{2}\right]>0
$$

Using (2.8), the expression

$$
\left[v_{00}\left\{\eta\left(\frac{2}{g}+f\right)+(v-1) f\right\}-v a_{0}^{2}\right]
$$

simplified to

$$
\begin{aligned}
& \frac{N}{\left[\sigma^{2}\{1+(N-1) \rho\}\right]^{2}} \times\left[\{c+(v-1)\} N \lambda_{4}\{1+(N-1) \rho\}\right. \\
& \left.-\{\eta+(v-1)\} \rho N^{2} \lambda_{2}^{2}-v N \lambda_{2}^{2}\right]
\end{aligned}
$$

Hence the non-singularity condition is

$$
\begin{aligned}
& {\left[\{c+(v-1)\} N \lambda_{4}\{1+(N-1) \rho\}\right.} \\
& \left.-\{\eta+(v-1)\} \rho N^{2} \lambda_{2}^{2}-v N \lambda_{2}^{2}\right]>0
\end{aligned}
$$

For any general second order slope rotatable designs (c.f. Hader and Park [11]), we have

$$
V\left(\hat{\beta}_{i i}\right)=\frac{1}{4} V\left(\hat{\beta}_{i j}\right) \text { i.e., } v^{i i . i i}=\frac{1}{4} v^{i j . i j}
$$

On simplification of (2.13), using (2.10), we get 


$$
\begin{aligned}
& \left(\frac{\eta\{1+(N-1) \rho\} 3 N \lambda_{4}-\rho N^{2} \lambda_{2}^{2}}{(1-\rho)}\right) \\
& {\left[4 N-\frac{\eta\{1+(N-1) \rho\} 3 N \lambda_{4}-\rho N^{2} \lambda_{2}^{2}}{\lambda_{4}\{1+(N-1) \rho\}}+v \frac{N \lambda_{2}^{2}(1-\rho)}{\lambda_{4}\{1+(N-1) \rho\}}-(v-2) \frac{\{1+(N-1) \rho\} N \lambda_{4}-\rho N^{2} \lambda_{2}^{2}}{\lambda_{4}\{1+(N-1) \rho\}}\right]} \\
& +\left(\frac{N\{1+(N-1) \rho\} N \lambda_{4}-\rho N^{2} \lambda_{2}^{2}}{(1-\rho)}\right)\left[4(v-2)+(v-1) \frac{\{1+(N-1) \rho\} N \lambda_{4}-\rho N^{2} \lambda_{2}^{2}}{N \lambda_{4}\{1+(N-1) \rho\}}\right] \\
& -N^{2} \lambda_{2}^{2}\left[4(v-1)+v \frac{\{1+(N-1) \rho\} N \lambda_{4}-\rho N^{2} \lambda_{2}^{2}}{N \lambda_{4}\{1+(N-1) \rho\}}\right]=0
\end{aligned}
$$

For $\rho=0$, Equation (2.15) reduces to (see Victorbabu and Narasimham [12])

$$
\lambda_{4}\left[v(5-c)-(c-3)^{2}\right]+\lambda_{2}^{2}[v(c-5)+4]=0
$$

On simplification of Equation (2.4) using Equation (2.6) and (2.13), we get

$$
\begin{aligned}
V\left(\frac{\partial \hat{y}_{x}}{\partial x_{i}}\right) & =e+4 x_{i}^{2}\left(\frac{g}{4}\right)+\sum_{j=1 ; j \neq i}^{v} x_{j}^{2} g \\
& =e+g \sum_{i=1}^{v} x_{i}^{2}=e+g d^{2}
\end{aligned}
$$

\section{New Method of Construction of RSOSRD Using Pairwise Balanced Designs}

Let there be an equi-replicated PBD with parameters ( $v$, $\left.b, r, k_{1}, k_{2}, \cdots, k_{p}, \lambda\right)$ and $k=\sup \left(k_{1}, k_{2}, \cdots, k_{p}\right), 2^{t(k)}$ denote a fractional replicate of $2^{k}$ in \pm levels, in which no interaction with less than five factors is confounded. $\left[1-\left(v, b, r, k_{1}, k_{2}, \cdots, k_{p}, \lambda\right)\right]$ denote the design points generated from the transpose of incidence matrix of PBD. $\left[1-\left(v, b, r, k_{1}, k_{2}, \cdots, k_{p}, \lambda\right)\right]$ are the $\mathrm{b} 2^{t(k)}$ design points generated from PBD (c.f. Raghavarao [17], pp. 298-300)), $n_{0}$ denote the number of central points and $( \pm \alpha, 0,0, \cdots, 0)$ $2^{1}$ denote the design points generated from $( \pm \alpha, 0,0, \cdots, 0)$ point set.

Here we start with usual SOSRD using PBD having " $n$ " (where $n=b 2^{t(k)}+2 v$ ) non-central design points involving $v$-factors. For this n-non-central design points we add $(n+1)\left(n+n_{0}=m\right.$ say $)$ central points in the following way.

One central point is placed in between each pair of non-central design points in the sequence, resulting thereby in $(n-1)$ such central points. The other two central points are placed one at the beginning and one at the end.

If the number of central points of the usual SOSRD with which we started is greater than $(n+1)$, the remaining central points are placed in any manner, if the number is less, we need to include the requisite number of additional central points. Here we examine the non-sin- gularity condition for the newly constructed design.

Let $N\left(N=b 2^{t(k)}+2 v+n_{0}\right)$ be the number of design points of an SOSRD using PBD with which we started. Out of $N$, let $\mathrm{n}$ be the number of non-central design points and $\mathrm{n}_{0}$ be the number of central points. i.e., $N=n$ $+n_{0}$. Let $\mathrm{N}_{1}$ be the number of design points of the newly constructed RSOSRD using PBD, where $N_{1}=n+m>N$. For the SOSRD using PBD with which we started, the following are the moment relations:

Theorem (3.1):

If $\left(v, b, r, k_{1}, k_{2}, \cdots, k_{p}, \lambda\right)$ is an equi-replicate PB design and $k=\sup \left(k_{1}, k_{2}, \cdots, k_{p}\right) 2^{t(k)}$ denotes a resolution $\mathrm{V}$ fractional replicate of of $2^{k}$ in \pm levels and $\mathrm{n}_{0}$ is the number of central points, then the design points, [1- $(v, b$, $\left.\left.r, k_{1}, k_{2}, \cdots, k_{p}, \lambda\right)\right] \times 2^{t(k)} U(\alpha, 0,0, \cdots, 0) \times 2^{t(k)} U(m)$ give a $v$-dimensional RSOSRD in $N_{1}=b 2^{t(k)}+2 v+m$ (where $m=n+n_{0}$ ) design points, where $\alpha^{2}$ is a positive real root (if it exists) of the biquadratic equation,

$$
\begin{aligned}
& \left(8 v-4 N_{1}\right) \alpha^{8}+8 v r 2^{t(k)}+\left[2 v r^{2} 2^{2 t(k)}\right. \\
& \left.+\left\{((12-2 v) \lambda-4 r) N_{1}+(16 \lambda-20 v \lambda+4 v r)\right\} 2^{t(k)}\right] \alpha^{4} \\
& +\left[4 v r^{2}+(16 v-20 v) r \lambda\right] 2^{2 t(k)} \alpha^{2} \\
& +\left[(5 v-9) \lambda^{2}+(6-v) r \lambda-r^{2}\right] N_{1} 2^{2 t(k)} \\
& +(v r+4 \lambda-5 v \lambda) r^{2} 2^{3 t(k)}=0 .
\end{aligned}
$$

If at least one positive real root of $\alpha^{2}$ exists in (3.1) then the design exists.

$c$ can be obtained from

$$
c=\frac{r 2^{t(k)}+2 \alpha^{4}}{2^{t(v)}} .
$$

Proof: For the design points generated from the PBD, simple symmetry conditions 1), 2), 2 of (2.7) are true. Condition 1) of (2.7) is true obviously. Conditions 2) and $3)$ of (2.7) are true as follows.

2) a) $\sum_{u=1}^{N_{1}} x_{i u}^{2}=r 2^{t(k)}+2 \alpha^{2}=$ constant $=N_{1} \lambda_{2}$, 


$$
\begin{aligned}
& 1 \leq i \leq v ; \\
& \text { b) } \sum_{u=1}^{N_{1}} x_{i u}^{4}=r 2^{t(k)}+2 \alpha^{4}=\text { constant }=c N_{1} \lambda_{4}, \\
& 1 \leq i \leq v ; \\
& \text { 3) } \sum_{u=1}^{N_{1}} x_{i u}^{2} x_{j u}^{2}=\lambda 2^{t(k)}=\text { constant }=N_{1} \lambda_{4}, \\
& 1 \leq i \neq j \leq v
\end{aligned}
$$

From 2) b) and 3) of (3.3), we get c given in (3.2). Substituting for $\lambda_{2}, \lambda_{4}$ and $\mathrm{c}$ in (2.16), and on simplification we get the fourth degree equation in $\alpha^{2}$ given in (3.1)

Corollary: If $k_{1}=k_{2}=\cdots=k_{p}=k$, then Theorem 3.1 reduces to the method of construction of RSOSRD using BIBD.

The RSOSRD using pairwise balanced designs values " $\alpha$ " and the variances of estimated slopes for the factors $6 \leq v \leq 15$ are given in Appendix.

Example: We illustrate the use of Theorem 3.1 by constructing a RSOSRD for 6-factors with the help of the PB design $\left(v=6, b=7, r=3, k_{1}=3, k_{2}=2, \lambda=1\right)$.

$[1-(6,7,3,3,2,1)] \times 2^{3} \mathrm{U}(\alpha, 0,0, \cdots, 0) \times 2^{1} U(m=$ 69) will give a RSOSRD in $N_{1}=137$ design points for six factors. From (3.3), we have

$$
\begin{aligned}
& \text { 2) a) } \sum_{u=1}^{N_{1}} x_{i u}^{2}=24+2 \alpha^{2}=N_{1} \lambda_{2}, 1 \leq i \leq v ; \\
& \text { b) } \sum_{u=1}^{N_{1}} x_{i u}^{4}=24+2 \alpha^{4}=c N_{1} \lambda_{4}, 1 \leq i \leq v ; \\
& \text { 3) } \sum_{u=1}^{N_{1}} x_{i u}^{2} x_{j u}^{2}=8=N_{1} \lambda_{4}, 1 \leq i \neq j \leq v ;
\end{aligned}
$$

Substituting for $\lambda_{2}, \lambda_{4}$ and $\mathrm{c}$ in (2.16), we get the following biquadratic equation.

$$
500 \alpha^{8}-1152 \alpha^{6}+6496 \alpha^{4}+6144 \alpha^{2}-68352=0 .
$$

(3.5) has only one positive real root $\alpha^{2}=2.7093$.

It may be pointed out here that this RSOSRD using PBD has only 137 design points for 6-factors, where as the corresponding RSOSRD obtained using a BIB design ( $v=6, b=10, r=5, k=3, \lambda=2$ ) needs 185 design points. Thus the new method sometimes leads to RSO-SRD using PBD with lesser number of design points than the RSOSRD obtained through BIB designs.

The Appendix gives the appropriate robust slope rotatability values of the parameter " $\alpha$ " for designs using a PBD, star points and for different number of central points and also variances and covariances of the factors for $6 \leq v \leq 15$.

\section{Acknowledgements}

The authors are thankful to the referee and the editor for the valuable suggestions which helped in improving the quality of the paper.

\section{REFERENCES}

[1] G. E. P. Box and J. S. Hunter, "Multifactor Experimental Designs for Exploring Response Surfaces," The Annals of Mathematical Statistics, Vol. 28, No. 1, 1957, pp. 195241. doi:10.1214/aoms/1177707047

[2] M. N. Das and V. L. Narasimham, "Construction of Rotatable Designs through Balanced Incomplete Block Designs," Annals of Mathematical Statistics, Vol. 33, No. 4, 1962, pp. 1421-1439.

[3] B. N. Tyagi, "On the Construction of Second Order and Third Order Rotatable Designs through Pairwise Balanced Designs and Doubly Balanced Designs," Calcutta Statistical Association Bulletin, Vol. 13, 1964, pp. 150162.

[4] R. N. Panda and R. N. Das, "First Order Rotatable Designs with Correlated Errors," Calcutta Statistical Association Bulletin, Vol. 44, 1994, pp. 83-101.

[5] R. N. Das, "Robust Second Order Rotatable Designs. PartI RSORD," Calcutta Statistical Association Bulletin, Vol. 47, 1997, pp. 199-214.

[6] R. N. Das, "Robust Second Order Rotatable Designs. PartII RSORD," Calcutta Statistical Association Bulletin, Vol. 49, 1999, pp. 65-76.

[7] K. Rajyalakshmi and B. Re. Victorbabu, "Robust Second Order Rotatable Central Composite Designs," JP Journal of Fundamental and Applied Statistics, Vol. 1, No. 2, 2011, pp. 85-102.

[8] B. Re. Victorbabu and K. Rajyalakshmi, "A New Method of Construction of Robust Second Order Rotatable Designs Using Balanced Incomplete Block Designs," Open Journal of Statistics, Vol. 2, No. 1, 2012, pp. 39-47.

[9] S. H. Park, "A Class of Multifactor Designs for Estimating the Slope of Response Surfaces," Technometrics, Vol. 29, No. 4, 1987, pp. 449-453.

[10] V. N. Murty and W. J. Studden, "Optimal Designs for Estimating the Slope of a Polynomial Regression," Journal of the American Statistical Association, Vol. 67, No. 340, 1972, pp. 869-873.

[11] R. J. Hader and S. H. Park, "Slope-Rotatable Central Composite Designs," Technometrics, Vol. 20, No. 4, 1978, pp. 413-417.

[12] B. Re. Victorbabu and V. L. Narasimham, "Construction of Second Order Slope Rotatable Designs through Balanced Incomplete Block Designs," Communications in Statistics-Theory and Methods, Vol. 20, No. 8, 1991, pp. 2467-2478. doi:10.1080/03610929108830644

[13] B. Re. Victorbabu and V. L. Narasimham, "Construction of Second Order Slope Rotatable Designs Using Pairwise Balanced Designs," Journal of the Indian Society of Agricultural Statistics, Vol. 45, No. 2, 1993, pp. 200-205.

[14] R. N. Das, "Slope Rotatability with Correlated Errors," Calcutta Statistical Association Bulletin, Vol. 54, 2003, pp. 57-70. 
[15] B. Re. Victorbabu and K. Rajyalakshmi, "Robust Slope Rotatable Central Composite Designs," Paper Submitted for the Possible Publication, 2012.

[16] B. Re. Victorbabu and K. Rajyalakshmi, "Robust Second Order Slope Rotatable Designs Using Balanced Incom- plete Block Designs," Paper Submitted for the Possible Publication, 2012.

[17] D. Raghavarao, "Constructions and Combinatorial Problems in Design of Experiments," John Wiley and Sons, New York, 1971. 


\section{Appendix}

\section{The Variance of Estimated Derivatives Slopes) for the Factors $6 \leq \boldsymbol{v} \leq \mathbf{1 5}$}

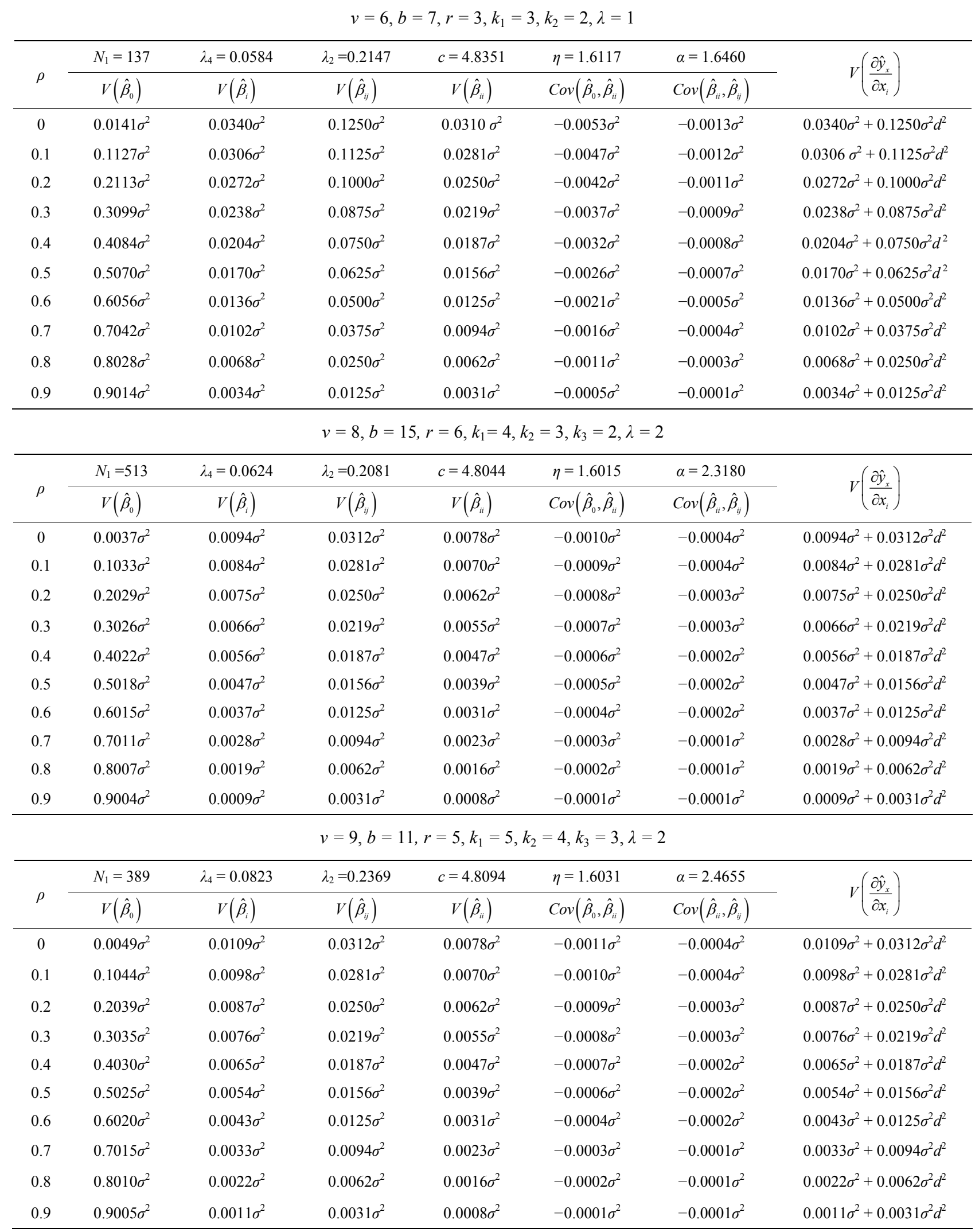


$v=10, b=11, r=5, k_{1}=5, k_{2}=4, \lambda=2$

\begin{tabular}{|c|c|c|c|c|c|c|c|}
\hline \multirow{2}{*}{$\rho$} & $N_{1}=393$ & $\lambda_{4}=0.0814$ & $\lambda_{2}=0.2345$ & $c=4.8162$ & $\eta=1.6054$ & $\alpha=2.4673$ & \multirow{2}{*}{$V\left(\frac{\partial \hat{y}_{x}}{\partial x_{i}}\right)$} \\
\hline & $V\left(\hat{\beta}_{0}\right)$ & $V\left(\hat{\beta}_{i}\right)$ & $V\left(\hat{\beta}_{i j}\right)$ & $V\left(\hat{\beta}_{i i}\right)$ & $\operatorname{Cov}\left(\hat{\beta}_{0}, \hat{\beta}_{i i}\right)$ & $\operatorname{Cov}\left(\hat{\beta}_{i i}, \hat{\beta}_{i j}\right)$ & \\
\hline 0 & $0.0050 \sigma^{2}$ & $0.0109 \sigma^{2}$ & $0.0313 \sigma^{2}$ & $0.0078 \sigma^{2}$ & $-0.0010 \sigma^{2}$ & $-0.0004 \sigma^{2}$ & $0.0109 \sigma^{2}+0.0313 \sigma^{2} d^{2}$ \\
\hline 0.1 & $0.1045 \sigma^{2}$ & $0.0098 \sigma^{2}$ & $0.0281 \sigma^{2}$ & $0.0070 \sigma^{2}$ & $-0.0009 \sigma^{2}$ & $-0.0003 \sigma^{2}$ & $0.0098 \sigma^{2}+0.0281 \sigma^{2} d^{2}$ \\
\hline 0.2 & $0.2040 \sigma^{2}$ & $0.0087 \sigma^{2}$ & $0.0250 \sigma^{2}$ & $0.0063 \sigma^{2}$ & $-0.0008 \sigma^{2}$ & $-0.0003 \sigma^{2}$ & $0.0087 \sigma^{2}+0.0250 \sigma^{2} d^{2}$ \\
\hline 0.3 & $0.3035 \sigma^{2}$ & $0.0076 \sigma^{2}$ & $0.0219 \sigma^{2}$ & $0.0055 \sigma^{2}$ & $-0.0007 \sigma^{2}$ & $-0.0003 \sigma^{2}$ & $0.0076 \sigma^{2}+0.0219 \sigma^{2} d^{2}$ \\
\hline 0.4 & $0.4030 \sigma^{2}$ & $0.0065 \sigma^{2}$ & $0.0188 \sigma^{2}$ & $0.0047 \sigma^{2}$ & $-0.0006 \sigma^{2}$ & $-0.0002 \sigma^{2}$ & $0.0065 \sigma^{2}+0.0188 \sigma^{2} d^{2}$ \\
\hline 0.5 & $0.5025 \sigma^{2}$ & $0.0054 \sigma^{2}$ & $0.0156 \sigma^{2}$ & $0.0039 \sigma^{2}$ & $-0.0005 \sigma^{2}$ & $-0.0002 \sigma^{2}$ & $0.0054 \sigma^{2}+0.0156 \sigma^{2} d^{2}$ \\
\hline 0.6 & $0.6020 \sigma^{2}$ & $0.0043 \sigma^{2}$ & $0.0125 \sigma^{2}$ & $0.0031 \sigma^{2}$ & $-0.0004 \sigma^{2}$ & $-0.0002 \sigma^{2}$ & $0.0043 \sigma^{2}+0.0125 \sigma^{2} d^{2}$ \\
\hline 0.7 & $0.7015 \sigma^{2}$ & $0.0033 \sigma^{2}$ & $0.0094 \sigma^{2}$ & $0.0023 \sigma^{2}$ & $-0.0003 \sigma^{2}$ & $-0.0001 \sigma^{2}$ & $0.0033 \sigma^{2}+0.0094 \sigma^{2} d^{2}$ \\
\hline 0.8 & $0.8010 \sigma^{2}$ & $0.0022 \sigma^{2}$ & $0.0063 \sigma^{2}$ & $0.0016 \sigma^{2}$ & $-0.0002 \sigma^{2}$ & $-0.0001 \sigma^{2}$ & $0.0022 \sigma^{2}+0.0063 \sigma^{2} d^{2}$ \\
\hline 0.9 & $0.9005 \sigma^{2}$ & $0.0011 \sigma^{2}$ & $0.0031 \sigma^{2}$ & $0.0008 \sigma^{2}$ & $-0.0001 \sigma^{2}$ & $-0.0001 \sigma^{2}$ & $0.0011 \sigma^{2}+0.0031 \sigma^{2} d^{2}$ \\
\hline \multicolumn{8}{|c|}{$v=12, b=16, r=6, k_{1}=6, k_{2}=5, k_{3}=4, k_{4}=3, \lambda=2$} \\
\hline \multirow[b]{2}{*}{$\rho$} & $N_{1}=1073$ & $\lambda_{4}=0.0596$ & $\lambda_{2}=0.1932$ & $c=4.8199$ & $\eta=1.6066$ & $\alpha=2.7625$ & \multirow{2}{*}{$V\left(\frac{\partial \hat{y}_{x}}{\partial x_{i}}\right)$} \\
\hline & $V\left(\hat{\beta}_{0}\right)$ & $V\left(\hat{\beta}_{i}\right)$ & $V\left(\hat{\beta}_{i j}\right)$ & $V\left(\hat{\beta}_{i i}\right)$ & $\operatorname{Cov}\left(\hat{\beta}_{0}, \hat{\beta}_{i i}\right)$ & $\operatorname{Cov}\left(\hat{\beta}_{i i}, \hat{\beta}_{i j}\right)$ & \\
\hline 0 & $0.0018 \sigma^{2}$ & $0.0048 \sigma^{2}$ & $0.0156 \sigma^{2}$ & $0.0039 \sigma^{2}$ & $-0.0004 \sigma^{2}$ & $-0.0002 \sigma^{2}$ & $0.0048 \sigma^{2}+0.0156 \sigma^{2} d^{2}$ \\
\hline 0.1 & $0.1016 \sigma^{2}$ & $0.0043 \sigma^{2}$ & $0.0141 \sigma^{2}$ & $0.0035 \sigma^{2}$ & $-0.0003 \sigma^{2}$ & $-0.0002 \sigma^{2}$ & $0.0043 \sigma^{2}+0.0141 \sigma^{2} d^{2}$ \\
\hline 0.2 & $0.2014 \sigma^{2}$ & $0.0039 \sigma^{2}$ & $0.0125 \sigma^{2}$ & $0.0031 \sigma^{2}$ & $-0.0003 \sigma^{2}$ & $-0.0001 \sigma^{2}$ & $0.0039 \sigma^{2}+0.0125 \sigma^{2} d^{2}$ \\
\hline 0.3 & $0.3012 \sigma^{2}$ & $0.0034 \sigma^{2}$ & $0.0109 \sigma^{2}$ & $0.0027 \sigma^{2}$ & $-0.0003 \sigma^{2}$ & $-0.0001 \sigma^{2}$ & $0.0039 \sigma^{2}+0.0109 \sigma^{2} d^{2}$ \\
\hline 0.4 & $0.4011 \sigma^{2}$ & $0.0029 \sigma^{2}$ & $0.0094 \sigma^{2}$ & $0.0023 \sigma^{2}$ & $-0.0002 \sigma^{2}$ & $-0.0001 \sigma^{2}$ & $0.0029 \sigma^{2}+0.0094 \sigma^{2} d^{2}$ \\
\hline 0.5 & $0.5009 \sigma^{2}$ & $0.0024 \sigma^{2}$ & $0.0078 \sigma^{2}$ & $0.0020 \sigma^{2}$ & $-0.0002 \sigma^{2}$ & $-0.0001 \sigma^{2}$ & $0.0024 \sigma^{2}+0.0078 \sigma^{2} d^{2}$ \\
\hline 0.6 & $0.6007 \sigma^{2}$ & $0.0019 \sigma^{2}$ & $0.0063 \sigma^{2}$ & $0.0016 \sigma^{2}$ & $-0.0001 \sigma^{2}$ & $-0.0001 \sigma^{2}$ & $0.0019 \sigma^{2}+0.0063 \sigma^{2} d^{2}$ \\
\hline 0.7 & $0.7005 \sigma^{2}$ & $0.0014 \sigma^{2}$ & $0.0047 \sigma^{2}$ & $0.0012 \sigma^{2}$ & $-0.0001 \sigma^{2}$ & $-0.0001 \sigma^{2}$ & $0.0014 \sigma^{2}+0.0047 \sigma^{2} d^{2}$ \\
\hline 0.8 & $0.8004 \sigma^{2}$ & $0.0010 \sigma^{2}$ & $0.0031 \sigma^{2}$ & $0.0008 \sigma^{2}$ & $-0.0001 \sigma^{2}$ & $-0.0001 \sigma^{2}$ & $0.0010 \sigma^{2}+0.0031 \sigma^{2} d^{2}$ \\
\hline 0.9 & $0.9002 \sigma^{2}$ & $0.0005 \sigma^{2}$ & $0.0016 \sigma^{2}$ & $0.0004 \sigma^{2}$ & $-0.0001 \sigma^{2}$ & $-0.0001 \sigma^{2}$ & $0.0005 \sigma^{2}+0.0016 \sigma^{2} d^{2}$ \\
\hline \multicolumn{8}{|c|}{$\left.v=13, b=16, r=6, k_{1}=6, k_{2}=5, k_{3}=4, k_{4}=3, \lambda=2\right)$} \\
\hline \multirow[b]{2}{*}{$\rho$} & $N_{1}=1077$ & $\lambda_{4}=0.0594$ & $\lambda_{2}=0.1925$ & $c=4.8273$ & $\eta=1.6091$ & $\alpha=2.7653$ & \multirow{2}{*}{$V\left(\frac{\partial \hat{y}_{x}}{\partial x_{i}}\right)$} \\
\hline & $V\left(\hat{\beta}_{0}\right)$ & $V\left(\hat{\beta}_{i}\right)$ & $V\left(\hat{\beta}_{i j}\right)$ & $V\left(\hat{\beta}_{i i}\right)$ & $\operatorname{Cov}\left(\hat{\beta}_{0}, \hat{\beta}_{i i}\right)$ & $\operatorname{Cov}\left(\hat{\beta}_{i i}, \hat{\beta}_{i j}\right)$ & \\
\hline 0 & $0.0018 \sigma^{2}$ & $0.0048 \sigma^{2}$ & $0.0156 \sigma^{2}$ & $0.0039 \sigma^{2}$ & $-0.0003 \sigma^{2}$ & $-0.0002 \sigma^{2}$ & $0.0048 \sigma^{2}+0.0156 \sigma^{2} d^{2}$ \\
\hline 0.1 & $0.1016 \sigma^{2}$ & $0.0043 \sigma^{2}$ & $0.0141 \sigma^{2}$ & $0.0035 \sigma^{2}$ & $-0.0003 \sigma^{2}$ & $-0.0002 \sigma^{2}$ & $0.0043 \sigma^{2}+0.0141 \sigma^{2} d^{2}$ \\
\hline 0.2 & $0.2014 \sigma^{2}$ & $0.0039 \sigma^{2}$ & $0.0125 \sigma^{2}$ & $0.0031 \sigma^{2}$ & $-0.0003 \sigma^{2}$ & $-0.0001 \sigma^{2}$ & $0.0039 \sigma^{2}+0.0125 \sigma^{2} d^{2}$ \\
\hline 0.3 & $0.3013 \sigma^{2}$ & $0.0034 \sigma^{2}$ & $0.0109 \sigma^{2}$ & $0.0027 \sigma^{2}$ & $-0.0002 \sigma^{2}$ & $-0.0001 \sigma^{2}$ & $0.0034 \sigma^{2}+0.0109 \sigma^{2} d^{2}$ \\
\hline 0.4 & $0.4011 \sigma^{2}$ & $0.0029 \sigma^{2}$ & $0.0094 \sigma^{2}$ & $0.0023 \sigma^{2}$ & $-0.0002 \sigma^{2}$ & $-0.0001 \sigma^{2}$ & $0.0029 \sigma^{2}+0.0094 \sigma^{2} d^{2}$ \\
\hline 0.5 & $0.5009 \sigma^{2}$ & $0.0024 \sigma^{2}$ & $0.0078 \sigma^{2}$ & $0.0020 \sigma^{2}$ & $-0.0002 \sigma^{2}$ & $-0.0001 \sigma^{2}$ & $0.0024 \sigma^{2}+0.0078 \sigma^{2} d^{2}$ \\
\hline 0.6 & $0.6007 \sigma^{2}$ & $0.0019 \sigma^{2}$ & $0.0063 \sigma^{2}$ & $0.0016 \sigma^{2}$ & $-0.0001 \sigma^{2}$ & $-0.0001 \sigma^{2}$ & $0.0019 \sigma^{2}+0.0063 \sigma^{2} d^{2}$ \\
\hline 0.7 & $0.7005 \sigma^{2}$ & $0.0014 \sigma^{2}$ & $0.0047 \sigma^{2}$ & $0.0012 \sigma^{2}$ & $-0.0001 \sigma^{2}$ & $-0.0001 \sigma^{2}$ & $0.0014 \sigma^{2}+0.0047 \sigma^{2} d^{2}$ \\
\hline 0.8 & $0.8004 \sigma^{2}$ & $0.0010 \sigma^{2}$ & $0.0031 \sigma^{2}$ & $0.0008 \sigma^{2}$ & $-0.0001 \sigma^{2}$ & $-0.0001 \sigma^{2}$ & $0.0010 \sigma^{2}+0.0031 \sigma^{2} d^{2}$ \\
\hline 0.9 & $0.9002 \sigma^{2}$ & $0.0005 \sigma^{2}$ & $0.0016 \sigma^{2}$ & $0.0004 \sigma^{2}$ & $-0.0001 \sigma^{2}$ & $-0.0001 \sigma^{2}$ & $0.0005 \sigma^{2}+0.0016 \sigma^{2} d^{2}$ \\
\hline
\end{tabular}


$v=14, b=16, r=6, k_{1}=6, k_{2}=5, k_{3}=4, \lambda=2$

\begin{tabular}{|c|c|c|c|c|c|c|c|}
\hline \multirow[b]{2}{*}{$\rho$} & $N_{1}=1081$ & $\lambda_{4}=0.0592$ & $\lambda_{2}=0.1918$ & $c=4.8342$ & $\eta=1.6114$ & $\alpha=2.7679$ & \multirow{2}{*}{$V\left(\frac{\partial \hat{y}_{x}}{\partial x_{i}}\right)$} \\
\hline & $V\left(\hat{\beta}_{0}\right)$ & $V\left(\hat{\beta}_{i}\right)$ & $V\left(\hat{\beta}_{i j}\right)$ & $V\left(\hat{\beta}_{i i}\right)$ & $\operatorname{Cov}\left(\hat{\beta}_{0}, \hat{\beta}_{i i}\right)$ & $\operatorname{Cov}\left(\hat{\beta}_{i i}, \hat{\beta}_{i j}\right)$ & \\
\hline 0 & $0.0018 \sigma^{2}$ & $0.0048 \sigma^{2}$ & $0.0156 \sigma^{2}$ & $0.0039 \sigma^{2}$ & $-0.0003 \sigma^{2}$ & $-0.0002 \sigma^{2}$ & $0.0048 \sigma^{2}+0.0156 \sigma^{2} d^{2}$ \\
\hline 0.1 & $0.1016 \sigma^{2}$ & $0.0043 \sigma^{2}$ & $0.0141 \sigma^{2}$ & $0.0035 \sigma^{2}$ & $-0.0003 \sigma^{2}$ & $-0.0002 \sigma^{2}$ & $0.0043 \sigma^{2}+0.0141 \sigma^{2} d^{2}$ \\
\hline 0.2 & $0.2014 \sigma^{2}$ & $0.0039 \sigma^{2}$ & $0.0125 \sigma^{2}$ & $0.0031 \sigma^{2}$ & $-0.0003 \sigma^{2}$ & $-0.0001 \sigma^{2}$ & $0.0039 \sigma^{2}+0.0125 \sigma^{2} d^{2}$ \\
\hline 0.3 & $0.3013 \sigma^{2}$ & $0.0034 \sigma^{2}$ & $0.0109 \sigma^{2}$ & $0.0027 \sigma^{2}$ & $-0.0002 \sigma^{2}$ & $-0.0001 \sigma^{2}$ & $0.0034 \sigma^{2}+0.0109 \sigma^{2} d^{2}$ \\
\hline 0.4 & $0.4011 \sigma^{2}$ & $0.0029 \sigma^{2}$ & $0.0094 \sigma^{2}$ & $0.0023 \sigma^{2}$ & $-0.0002 \sigma^{2}$ & $-0.0001 \sigma^{2}$ & $0.0029 \sigma^{2}+0.0094 \sigma^{2} d^{2}$ \\
\hline 0.5 & $0.5009 \sigma^{2}$ & $0.0024 \sigma^{2}$ & $0.0078 \sigma^{2}$ & $0.0020 \sigma^{2}$ & $-0.0002 \sigma^{2}$ & $-0.0001 \sigma^{2}$ & $0.0024 \sigma^{2}+0.0078 \sigma^{2} d^{2}$ \\
\hline 0.6 & $0.6007 \sigma^{2}$ & $0.0019 \sigma^{2}$ & $0.0063 \sigma^{2}$ & $0.0016 \sigma^{2}$ & $-0.0001 \sigma^{2}$ & $-0.0001 \sigma^{2}$ & $0.0019 \sigma^{2}+0.0063 \sigma^{2} d^{2}$ \\
\hline 0.7 & $0.7005 \sigma^{2}$ & $0.0014 \sigma^{2}$ & $0.0047 \sigma^{2}$ & $0.0012 \sigma^{2}$ & $-0.0001 \sigma^{2}$ & $-0.0001 \sigma^{2}$ & $0.0014 \sigma^{2}+0.0047 \sigma^{2} d^{2}$ \\
\hline 0.8 & $0.8004 \sigma^{2}$ & $0.0010 \sigma^{2}$ & $0.0031 \sigma^{2}$ & $0.0008 \sigma^{2}$ & $-0.0001 \sigma^{2}$ & $-0.0001 \sigma^{2}$ & $0.0010 \sigma^{2}+0.0031 \sigma^{2} d^{2}$ \\
\hline 0.9 & $0.9002 \sigma^{2}$ & $0.0005 \sigma^{2}$ & $0.0016 \sigma^{2}$ & $0.0004 \sigma^{2}$ & $-0.0001 \sigma^{2}$ & $-0.0001 \sigma^{2}$ & $0.0005 \sigma^{2}+0.0016 \sigma^{2} d^{2}$ \\
\hline \multicolumn{8}{|c|}{$v=15, b=16, r=6, k_{1}=6, k_{2}=5, \lambda=2$} \\
\hline \multirow[b]{2}{*}{$\rho$} & $N_{1}=1085$ & $\lambda_{4}=0.0590$ & $\lambda_{2}=0.1911$ & $c=4.8406$ & $\eta=1.6135$ & $\alpha=2.7703$ & \multirow{2}{*}{$V\left(\frac{\partial \hat{y}_{x}}{\partial x_{i}}\right)$} \\
\hline & $V\left(\hat{\beta}_{0}\right)$ & $V\left(\hat{\beta}_{i}\right)$ & $V\left(\hat{\beta}_{i j}\right)$ & $V\left(\hat{\beta}_{i i}\right)$ & $\operatorname{Cov}\left(\hat{\beta}_{0}, \hat{\beta}_{i i}\right)$ & $\operatorname{Cov}\left(\hat{\beta}_{i i}, \hat{\beta}_{i j}\right)$ & \\
\hline 0 & $0.0048 \sigma^{2}$ & $0.0156 \sigma^{2}$ & $0.0018 \sigma^{2}$ & $0.0039 \sigma^{2}$ & $-0.0003 \sigma^{2}$ & $-0.0002 \sigma^{2}$ & $0.0048 \sigma^{2}+0.0156 \sigma^{2} d^{2}$ \\
\hline 0.1 & $0.0043 \sigma^{2}$ & $0.0141 \sigma^{2}$ & $0.1016 \sigma^{2}$ & $0.0035 \sigma^{2}$ & $-0.0003 \sigma^{2}$ & $-0.0001 \sigma^{2}$ & $0.0043 \sigma^{2}+0.0141 \sigma^{2} d^{2}$ \\
\hline 0.2 & $0.0039 \sigma^{2}$ & $0.0125 \sigma^{2}$ & $0.2015 \sigma^{2}$ & $0.0031 \sigma^{2}$ & $-0.0002 \sigma^{2}$ & $-0.0001 \sigma^{2}$ & $0.0039 \sigma^{2}+0.0125 \sigma^{2} d^{2}$ \\
\hline 0.3 & $0.0034 \sigma^{2}$ & $0.0109 \sigma^{2}$ & $0.3013 \sigma^{2}$ & $0.0027 \sigma^{2}$ & $-0.0002 \sigma^{2}$ & $-0.0001 \sigma^{2}$ & $0.0034 \sigma^{2}+0.0109 \sigma^{2} d^{2}$ \\
\hline 0.4 & $0.0029 \sigma^{2}$ & $0.0094 \sigma^{2}$ & $0.4011 \sigma^{2}$ & $0.0023 \sigma^{2}$ & $-0.0002 \sigma^{2}$ & $-0.0001 \sigma^{2}$ & $0.0029 \sigma^{2}+0.0094 \sigma^{2} d^{2}$ \\
\hline 0.5 & $0.0024 \sigma^{2}$ & $0.0078 \sigma^{2}$ & $0.5009 \sigma^{2}$ & $0.0020 \sigma^{2}$ & $-0.0002 \sigma^{2}$ & $-0.0001 \sigma^{2}$ & $0.0024 \sigma^{2}+0.0078 \sigma^{2} d^{2}$ \\
\hline 0.6 & $0.0019 \sigma^{2}$ & $0.0062 \sigma^{2}$ & $0.6007 \sigma^{2}$ & $0.0016 \sigma^{2}$ & $-0.0001 \sigma^{2}$ & $-0.0001 \sigma^{2}$ & $0.0019 \sigma^{2}+0.0062 \sigma^{2} d^{2}$ \\
\hline 0.7 & $0.0014 \sigma^{2}$ & $0.0047 \sigma^{2}$ & $0.7005 \sigma^{2}$ & $0.0012 \sigma^{2}$ & $-0.0001 \sigma^{2}$ & $-0.0001 \sigma^{2}$ & $0.0014 \sigma^{2}+0.0047 \sigma^{2} d^{2}$ \\
\hline 0.8 & $0.0010 \sigma^{2}$ & $0.0031 \sigma^{2}$ & $0.8004 \sigma^{2}$ & $0.0008 \sigma^{2}$ & $-0.0001 \sigma^{2}$ & $-0.0001 \sigma^{2}$ & $0.0010 \sigma^{2}+0.0031 \sigma^{2} d^{2}$ \\
\hline 0.9 & $0.0005 \sigma^{2}$ & $0.0016 \sigma^{2}$ & $0.9002 \sigma^{2}$ & $0.0004 \sigma^{2}$ & $-0.0001 \sigma^{2}$ & $-0.0001 \sigma^{2}$ & $0.0005 \sigma^{2}+0.0016 \sigma^{2} d^{2}$ \\
\hline
\end{tabular}

